\title{
Consumer's Demand and the Online Promotion of the e-service Quality through the Websites-An Empirical Study in China
}

\author{
Zhenfeng Wei \\ Zhejiang Industry \& Trade Vocational College \\ Address: No.717 Fudong Road, Wenzhou City, Zhejiang Province, China, 325003 \\ wzf456@163.com
}

\begin{abstract}
Over the past several years electronic commerce (e-commerce) has changed the business transaction way, and online service quality has a significant influence on many important aspects of e-commerce. However, there are few quantitative studies on the investigation of the online service quality evaluation focused on the market in China, which is one of the developing countries with the highest online population growth. This paper is an attempt to identify the main factors affecting the online service satisfaction of the e-commerce websites in China, and Fuzzy TOPSIS is employed to evaluate the eservice quality through internet for the online consumers. The results could be the guideline for the e-commerce companies in terms of improving their online services.
\end{abstract}

Keywords: online service, Fuzzy TOPSIS, empirical study, China

\section{Introduction}

In today's intensive competitive environments the design and delivery of innovative, flexible and effective service is of paramount importance for business success [1, 2]. As the development of the internet, there are increasingly online shoppers in the world involving in the related e-commerce activities. As the one of the developing countries, China has the highest online population growth rates of online shopping. The online service quality and satisfaction are becoming increasingly important as the e-commerce companies deliver an expanding array of services through the internet. Online service quality has a significant influence on many aspects of the e-commerce, which include the consumer trust on the e-commerce companies [3-5]; attitude toward e-shopping [6]; site loyalty intentions $[7,8]$; willingness to pay more $[9,10]$ and user online satisfaction $[10,7]$.

Online e-service has been recognized as one of the most important determinants of long-term performance and success for e-commerce retailers [11-14]. It is necessary for the e-commerce companies to identify customers' needs, wants, and improve the e-service quality [15].

The remainder of this study is organized as follows. Section 2 introduces the related literature about the online service quality. Following is a brief introduction about the Fuzzy TOPSIS which is employed in this research. Section 4 discusses the main factors that have an impact on e-service through the internet and identifies the current issues in terms of improving the online service quality of the top 15 B2C e-commerce websites in retailing market in China. Section 5 is an empirical analysis of evaluating the websites of these e-commerce retailers. In the last section, the related managerial implications and major challenges for these e-commerce retailers in promoting their e-service are identified in the last part of this research. 


\section{Literature Review}

Recent developments in terms of e-service have led researchers and practitioners to reevaluate certain traditional concepts, such as service quality and satisfaction, in the context of information technology. Online consumers actively participate in service delivery, supplying their own effort and time, and consumers also contribute by assuming part of the responsibility for service delivery. This aspect of online purchasing can affect perceptions of service quality and satisfaction [16]. Whereas traditional service quality is defined as a "consumer's judgment about anentity's overall excellence or superiority" [17], e-service quality represents "the extent to which a website facilitates efficient and effective shopping, purchasing and delivery" [17]. The relevant literature on e-service quality indicates that a website is not simply a utilitarian tool for finding information, making choices, and ordering a product or service [18].

A series of researches have been done in terms of the web site quality measurement [19, 8], online service quality evaluation [20-22,17,16], or e-retailing quality appraisal [13] are done. In general, these results derive from rigorous development efforts and focus on important characteristics pertaining to information or the system; few consider the service dimension of online services comprehensively [23-25]. The related research results are summarized in Table 1:

Table 1. Online Service Quality Scales in Prior Related Research

\begin{tabular}{|c|c|c|}
\hline Article & System related & Service related \\
\hline $\begin{array}{l}\text { Zeithaml et al. } \\
\text { [16] }\end{array}$ & $\begin{array}{l}\text { Access, ease of navigation, flexibility, reliability, } \\
\text { price knowledge, aesthetics, efficiency, } \\
\text { personalization, privacy. }\end{array}$ & $\begin{array}{l}\text { Responsiveness, } \\
\text { assurance }\end{array}$ \\
\hline $\begin{array}{l}\text { Francis and } \\
\text { White[26] }\end{array}$ & $\begin{array}{l}\text { Product attribute, functionality, ownership } \\
\text { conditions, security }\end{array}$ & $\begin{array}{l}\text { Delivery, customer } \\
\text { service }\end{array}$ \\
\hline $\begin{array}{l}\text { Loiacono et al. } \\
\text { [19] }\end{array}$ & $\begin{array}{l}\text { Appeal, response time, flow, image, operations, } \\
\text { better than alternatives, innovativeness, } \\
\text { interactivity, trust }\end{array}$ & \\
\hline $\begin{array}{l}\text { Barnes \& } \\
\text { Vidgen[27] }\end{array}$ & Usability, design & Empathy, trust \\
\hline $\begin{array}{l}\text { Wolfinbarger \& } \\
\text { Gilly[13] }\end{array}$ & Website design, privacy & $\begin{array}{l}\text { Fulfillment/reliability, } \\
\text { customer service }\end{array}$ \\
\hline $\begin{array}{l}\text { Parasuraman et } \\
\text { al.[17] }\end{array}$ & Efficiency, availability, privacy & Fulfillment \\
\hline $\begin{array}{l}\text { Parasuraman et } \\
\text { al.[17] }\end{array}$ & & $\begin{array}{l}\text { Compensation, } \\
\text { responsiveness } \\
\text { contract }\end{array}$ \\
\hline Bauer et al. [21] & Reliability, process, functionality/design & $\begin{array}{l}\text { Responsiveness, } \\
\text { enjoyment }\end{array}$ \\
\hline $\begin{array}{l}\text { Yoo \& Donthu } \\
{[8]}\end{array}$ & Ease of use, aesthetic design, reliability, tangibles & Responsiveness \\
\hline $\begin{array}{l}\text { Aldwani \& } \\
\text { Palvia[28] }\end{array}$ & $\begin{array}{l}\text { Technical adequacy, specific content, content } \\
\text { quality, web appearance }\end{array}$ & \\
\hline Janda et al. [29] & Access, security, information & Sensation \\
\hline $\begin{array}{l}\text { Ranganathan \& } \\
\text { Ganapathy [30] }\end{array}$ & Information content, design, security, privacy & \\
\hline $\begin{array}{l}\text { Yang \& Jun } \\
{[31]}\end{array}$ & $\begin{array}{l}\text { Reliability, access, ease of use, personalization, } \\
\text { security }\end{array}$ & Responsiveness \\
\hline Cai \& Jun [32] & Website design/content & $\begin{array}{l}\text { Trustworthiness, } \\
\text { prompt/reliable } \\
\text { service, } \\
\text { communication }\end{array}$ \\
\hline $\begin{array}{l}\text { Gounaris \& } \\
\text { Dimitriadis [1] }\end{array}$ & & $\begin{array}{l}\text { Customer care and } \\
\text { risk reduction benefit, }\end{array}$ \\
\hline
\end{tabular}


Jun et al. [33]

Ease of use, attentiveness, access, security, credibility

Kim \& Stoel

[34]

Lee \& Lin [9]

Web appearance, entertainment, information fit-totask, transaction capacity

Parasuraman et

al. [17]

Yang et al. [35]

Collier and

Bienstock [36]

Ibrahim et al.

Cristobal et al.

[10]

Ho \& Lee [7] Information quality, security, website functionality

Sohn \&

Tadisina[38]

Wang et al. $[39]$

Trust, ease of use, website content and

functionality, reliability

Website design, reliability, personalization

Efficiency, system availability, privacy

sability, usefulness of content, adequacy of information, accessibility

Functionality, information accuracy, design, privacy, ease of use, order condition, order accuracy, procedural fairness, outcome fairness Convenience/accuracy, accessibility/reliability, good queue management, personalization

Ding et al. $[40$

Reliability, competence, ease of use, product portfolio, security

Hamed et al. [25]

Intangibility, process nature, heterogeneity, inseparability which is the simultaneousness of consumption, production and marketing, perishability, ownership, interactive nature information benefit, interaction facilitation Reliable/prompt responses

Response time, trust

Responsiveness, trust Fulfillment

Interaction

Timeliness, interactive fairness

Friendly/responsive customer service, targeted customer service Customer service

Customer relationships, responsiveness Customized communication, speed of delivery Responsiveness, satisfaction Service convenience, customer service, fulfillment Self-service, nonrival

\section{Methodology}

\subsection{Fuzzy Sets and Fuzzy Numbers}

Definition 1: A Fuzzy set $\tilde{a}$ in a universe of discourse $X$ is characterized by a membership function $\mu_{\tilde{a}}(x)$ which associates with each element $x$ in $X$, a real number in the interval $[0,1]$. The function of $\mu_{\tilde{a}}(x)$ is termed the grade of membership of $x$ in $\tilde{a}$. The present study uses triangular Fuzzy numbers. $\tilde{a}$ can be defined by a triplet $\left(a_{1}, a_{2}, a_{3}\right)$. Its conceptual schema and mathematical form are shown as below:

$$
\mu_{\tilde{a}}(x) \begin{cases}0 & x \leq a \\ \frac{x-a_{1}}{a_{2}-a_{1}} & a_{1} \prec x \leq a_{2} \\ \frac{a_{3}-x}{a_{3}-a_{2}} & a_{2} \prec x \leq a_{3} \\ 1 & x \succ a_{3}\end{cases}
$$


Definition 2: Let $\tilde{a}=\left(a_{1}, a_{2}, a_{3}\right)$ and $\tilde{b}=\left(b_{1}, b_{2}, b_{3}\right)$ be two triangular fuzzy numbers. According to Wang (2009), a distance measure function $(\tilde{a}, \tilde{b})$ can be defined as below:

$$
d(\tilde{a}, \tilde{b})=\sqrt{\frac{1}{3}\left[\left(a_{1}-b_{1}\right)^{2}+\left(a_{2}-b_{2}\right)^{2}+\left(a_{3}-b_{3}\right)^{2}\right]}
$$

Definition 3: Let a triangular Fuzzy number $\tilde{a}$, then $\alpha$-cut defined as below:

$$
\tilde{a}_{\alpha}=\left[\left(a_{2}-a_{1}\right) \alpha+a_{1}, a_{3}-\left(a_{3}-a_{2}\right) \alpha\right]
$$

Definition 4: Let $\tilde{a}=\left(a_{1}, a_{2}, a_{3}\right), \tilde{b}=\left(b_{1}, b_{2}, b_{3}\right)$ be two triangular Fuzzy number and $\tilde{a}_{\alpha}, \tilde{b}_{\alpha}$ be $\alpha$-cut, $\tilde{a}$ and $\tilde{b}$, then the method is defined to calculate the divided between $\tilde{a}$ and $\tilde{b}$ as follows:

$$
\frac{\tilde{a}_{\alpha}}{\tilde{b}_{\alpha}}=\left[\frac{\left(a_{2}-a_{1}\right) \alpha+a_{1}}{-\left(b_{3}-b_{2}\right) \alpha+b_{3}}, \frac{-\left(a_{3}-a_{2}\right) \alpha+a_{3}}{\left(b_{2}-b_{1}\right) \alpha+b_{1}}\right]
$$

When $\alpha=0$,

$$
\frac{\tilde{a}_{0}}{\tilde{b}_{0}}=\left[\frac{a_{1}}{b_{3}}, \frac{a_{3}}{b_{1}}\right]
$$

When $\alpha=1$

$$
\begin{aligned}
& \frac{\tilde{a}_{1}}{\tilde{b}_{1}}=\left[\frac{\left(a_{2}-a_{1}\right)+a_{1}}{-\left(b_{3}-b_{2}\right)+b_{3}}, \frac{-\left(a_{3}-a_{2}\right)+a_{3}}{\left(b_{2}-b_{1}\right)+b_{1}}\right] \\
& \frac{\tilde{a}_{1}}{\tilde{b}_{1}}=\left[\frac{a_{2}}{b_{2}}, \frac{a_{2}}{b_{2}}\right]
\end{aligned}
$$

So the approximated value of $\tilde{a} / \tilde{b}$ will be

$$
\frac{\tilde{a}}{\tilde{b}}=\left[\frac{a_{1}}{b_{3}}, \frac{a_{2}}{b_{2}}, \frac{a_{3}}{b_{1}}\right]
$$

Definition 5: Assuming that both $\tilde{a}=\left(a_{1}, a_{2}, a_{3}\right)$ and $\tilde{b}=\left(b_{1}, b_{2}, b_{3}\right)$ are real numbers, the distance measurement $d(\tilde{a}, \tilde{b})$ is identical to the Euclidean distance.

The basic operations on Fuzzy triangular numbers are as follows:

For approximation of multiplication: $\tilde{a} \otimes \tilde{b}=\left(a_{1} \times b_{1}, a_{2} \times b_{2}, a_{3} \times b_{3}\right)$

For addition: $\tilde{a} \oplus \tilde{b}=\left(a_{1}+b_{1}, a_{2}+b_{2}, a_{3}+b_{3}\right)$

\subsection{Fuzzy Membership Function}

In the evaluating process, the weights expressed with the linguistic terms, represent the important degrees of criteria from experts via surveys on subjective assessments. These linguistic terms are categorized into very low (VL), low (L), medium (M), high (H) and very high $(\mathrm{VH})$. Assume that all linguistic terms can be transferred into triangular fuzzy numbers, and these fuzzy numbers are limited in $[0,1]$. As a rule of thumb, each rank is assigned an evenly spread membership function that has an interval of 0.30 or 0.25 .

Based on assumptions above, a transformation table can be found as shown in Table 2. Figure 1 illustrates the Fuzzy membership function. 
Table 2. Transformation for Fuzzy Membership Functions

\begin{tabular}{lcr}
\hline Rank & Sub-criteria grade & Membership function \\
\hline Very Low (VL) & 1 & $(0.00,0.10,0.25)$ \\
Low (L) & 2 & $(0.15,0.30,0.45)$ \\
Medium (M) & 3 & $(0.35,0.50,0.65)$ \\
High (H) & 4 & $(0.55,0.70,0.85)$ \\
Very High (VH) & 5 & $(0.75,0.90,1.00)$ \\
\hline
\end{tabular}

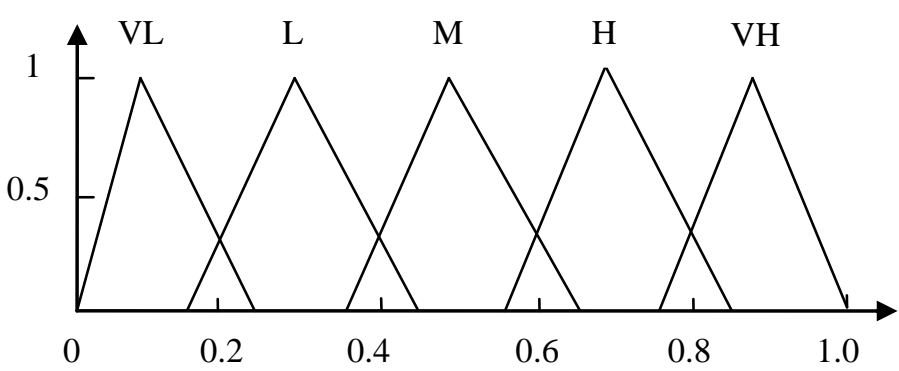

Figure 1. Fuzzy Triangular Membership Functions

\subsection{Fuzzy TOPSIS Model}

It is formulated that a Fuzzy Multiple Criteria Decision Making (FMCDM) problem about the comparative evaluation of the selected websites. The FMCDM problem can be concisely expressed in matrix format as follows:

$$
\begin{aligned}
& \begin{array}{lllll}
C_{1} & C_{2} & C_{3} & \cdots & C_{n}
\end{array} \\
& \begin{array}{l|lllll}
A_{1} & \left\lceil\tilde{x}_{11}\right. & \tilde{x}_{12} & \tilde{x}_{13} & \cdots & \tilde{x}_{1 n} \\
A_{2} & \tilde{x}_{21} & \tilde{x}_{22} & \tilde{x}_{23} & \cdots & \tilde{x}_{2 n}
\end{array} \mid \\
& \begin{array}{l|lllll}
A_{3} & \tilde{x}_{31} & \tilde{x}_{32} & \tilde{x}_{33} & \cdots & \tilde{x}_{3 n}
\end{array} \\
& A_{n}\left[\begin{array}{lllll}
\vdots & & & & \\
\tilde{x}_{n 1} & \tilde{x}_{n 1} & \tilde{x}_{n 1} & \cdots & \tilde{x}_{n 1}
\end{array}\right] \\
& \tilde{W}=\left[\tilde{w}_{1}, \tilde{w}_{2}, \cdots \tilde{w}_{n}\right]
\end{aligned}
$$

Where $x_{i j}, i=1,2, \cdots, m ; j=1,2, \cdots, n$ and $\tilde{w}_{j}, j=1,2, \cdots, n$ are linguistic triangular Fuzzy numbers, $\tilde{x}_{i j}=\left(a_{i j}, b_{i j}, c_{i j}\right)$ and $\tilde{w}_{j}=\left(a_{j 1}, b_{j 2}, c_{j 3}\right)$. The normalized Fuzzy decision matrix is denoted by $\tilde{R}=\left[\tilde{r}_{i j}\right]_{m \times n}$.

The weighted Fuzzy normalized decision matrix is shown as follows:

$$
\begin{aligned}
& V=\left[\begin{array}{ccccc}
\tilde{v}_{11} & \tilde{v}_{12} & \tilde{v}_{13} & \cdots & \tilde{v}_{1 n} \\
\tilde{v}_{21} & \tilde{v}_{22} & \tilde{v}_{23} & \cdots & \tilde{v}_{2 n} \\
\tilde{v}_{31} & \tilde{v}_{32} & \tilde{v}_{33} & \cdots & \tilde{v}_{3 n} \\
\vdots & & & & \\
\tilde{v}_{n 1} & \tilde{v}_{n 1} & \tilde{v}_{n 1} & \cdots & \tilde{v}_{n 1}
\end{array}\right] \\
& \left\lceil\mid \begin{array}{lllll}
\tilde{w}_{1} \tilde{r}_{11} & \tilde{w}_{2} \tilde{r}_{12} & \tilde{w}_{3} \tilde{r}_{13} & \cdots & \tilde{w}_{n} \tilde{r}_{1 n} \\
\tilde{w}_{1} \tilde{r}_{21} & \tilde{w}_{2} \tilde{r}_{22} & \tilde{w}_{3} \tilde{r}_{23} & \cdots & \tilde{w}_{n} \tilde{r}_{2 n} \\
\tilde{w}_{1} \tilde{r}_{31} & \tilde{w}_{2} \tilde{r}_{32} & \tilde{w}_{3} \tilde{r}_{33} & \cdots & \tilde{w}_{n} \tilde{r}_{3 n} \\
\vdots & & & & \\
\tilde{w}_{1} \tilde{r}_{m 1} & \tilde{w}_{2} \tilde{r}_{m 2} & \tilde{w}_{3} \tilde{r}_{m 3} & \cdots & \tilde{w}_{n} \tilde{r}_{m n}
\end{array}\right]
\end{aligned}
$$


Given the above Fuzzy theory, the proposed Fuzzy TOPSIS procedure is then defined as follows:

Step 1: choose the $x_{i j}, i=1,2, \cdots, m ; j=1,2, \cdots, n$ for alternatives with respect to criteria and $\tilde{w}_{j}, j=1,2, \cdots, n$ for the weight of the criteria.

Step 2: Construct the weighted normalized Fuzzy decision matrix $V$.

Step 3: Identify positive ideal $\left(A^{+}\right)$and negative ideal $\left(A^{-}\right)$solutions:

$$
\begin{aligned}
A^{+} & =\left\{\tilde{v}_{1}^{+}, \tilde{v}_{2}^{+}, \cdots \tilde{v}_{n}^{+}\right\} \\
& =\left\{\left(\max _{i} \tilde{v}_{i j} \mid i=1,2, \cdots, m\right), j=1,2, \cdots, n\right\} . \\
A^{-} & =\left\{\tilde{v}_{1}^{-}, \tilde{v}_{2}^{-}, \cdots \tilde{v}_{n}^{-}\right\} \\
& =\left\{\left(\min _{i} \tilde{v}_{i j} \mid i=1,2, \cdots, m\right), j=1,2, \cdots, n\right\} .
\end{aligned}
$$

Step4: Calculate separation measures. The distance of each alternative from $A^{+}$and $A^{-}$can be identified as follows:

$$
\begin{aligned}
& d_{i}^{+}=\frac{1}{n} \sum_{j=1}^{n} d\left(\tilde{v}_{i j}, \tilde{v}_{j}^{+}\right), i=1,2, \cdots, m \\
& d_{i}^{-}=\frac{1}{n} \sum_{j=1}^{n} d\left(\tilde{v}_{i j}, \tilde{v}_{j}^{-}\right), i=1,2, \cdots, m
\end{aligned}
$$

Step 5: Calculate the similarities to ideal solution:

$$
C C_{i}=\frac{d_{i}^{-}}{d_{i}^{+}+d_{i}^{-}}
$$

Step 6: Rank alternatives according to $C C_{i}$ in descending order (Yang and Hung, 2007).

\section{Data Collection and Results Analysis}

Considering the differences among $\mathrm{B} 2 \mathrm{C}, \mathrm{C} 2 \mathrm{C}$ and $\mathrm{B} 2 \mathrm{~B}$ e-commerce, and our research is focus on $\mathrm{B} 2 \mathrm{C}$ e-commerce websites in China, the top $15 \mathrm{~B} 2 \mathrm{C}$ e-commerce websites in retail market as shown in table 3 are selected based on the user coverage.

\section{Table 3. The Top 15 B2C e-commerce Websites in Retail Market in China}

\begin{tabular}{llc}
\hline No. & E-Commerce Website & No. of users (per million) \\
\hline$E_{1}$ & Tmall & 9010 \\
$E_{2}$ & Jingdong Mall & 6940 \\
$E_{3}$ & Tencent & 3930 \\
$E_{4}$ & Amazon & 3450 \\
$E_{5}$ & Handle group buying & 2580 \\
$E_{6}$ & Dangdang & 2160 \\
$E_{7}$ & Vancl & 2160 \\
$E_{8}$ & Full King & 1290 \\
$E_{9}$ & No.1 & 1050 \\
$E_{10}$ & F group buying & 770 \\
$E_{11}$ & Yixun & 760 \\
$E_{12}$ & Moonbasa & 700 \\
$E_{13}$ & Letao & 640 \\
$E_{14}$ & Newegg & 600 \\
$E_{15}$ & M18 & 570 \\
\hline
\end{tabular}

The specific original measures are listed in Table 4. The decision problem contains three levels: the highest level is the objective of the problem, while the criteria are listed in the second level, and the sub-criteria are listed in the third level. 
Table 4. The Measures of Online Service Quality

\begin{tabular}{|l|l|l|}
\hline Goal & Aspects & Criteria \\
\hline & & $C_{1}$ Efficiency \\
& & $C_{2}$ Ease of navigation \\
& & $C_{3}$ Flexibility \\
& & $C_{4}$ Reliability \\
Assessment & \multirow{4}{*}{$A_{1}$ System related } & $C_{5}$ Price knowledge \\
of online & & $C_{6}$ Aesthetics \\
service & & $C_{7}$ Personalization \\
quality & & $C_{8}$ Ownership conditions \\
& & $C_{9}$ Ease of use \\
\cline { 2 - 3 } & \multirow{3}{*}{$A_{2}$ Service related } & $C_{10}$ Speed \\
\hline & & $C_{11}$ Responsiveness \\
& & $C_{12}$ Assurance \\
& & $C_{13}$ Delivery \\
& & $C_{14}$ Customer service \\
\hline
\end{tabular}

The important degrees of the above sub-criteria weights are given with linguistic terms, as shown in Table 2, employed by four decision makers, as shown in Table 5.

Table 5. The Fuzzy Weights Given by Four Decision Makers

\begin{tabular}{lllll}
\hline Criteria & $\mathrm{DM}_{1}$ & $\mathrm{DM}_{2}$ & $\mathrm{DM}_{3}$ & $\mathrm{DM}_{4}$ \\
\hline$C_{1}$ & $\mathrm{H}$ & $\mathrm{M}$ & $\mathrm{H}$ & $\mathrm{VH}$ \\
$C_{2}$ & $\mathrm{VH}$ & $\mathrm{H}$ & $\mathrm{H}$ & $\mathrm{VH}$ \\
$C_{3}$ & $\mathrm{~L}$ & $\mathrm{M}$ & $\mathrm{M}$ & $\mathrm{M}$ \\
$C_{4}$ & $\mathrm{H}$ & $\mathrm{M}$ & $\mathrm{H}$ & $\mathrm{H}$ \\
$C_{5}$ & $\mathrm{H}$ & $\mathrm{M}$ & $\mathrm{H}$ & $\mathrm{H}$ \\
$C_{6}$ & $\mathrm{M}$ & $\mathrm{L}$ & $\mathrm{M}$ & $\mathrm{L}$ \\
$C_{7}$ & $\mathrm{~L}$ & $\mathrm{~L}$ & $\mathrm{~L}$ & $\mathrm{VL}$ \\
$C_{8}$ & $\mathrm{M}$ & $\mathrm{H}$ & $\mathrm{M}$ & $\mathrm{L}$ \\
$C_{9}$ & $\mathrm{M}$ & $\mathrm{L}$ & $\mathrm{M}$ & $\mathrm{L}$ \\
$C_{10}$ & $\mathrm{M}$ & $\mathrm{H}$ & $\mathrm{L}$ & $\mathrm{H}$ \\
$C_{11}$ & $\mathrm{~L}$ & $\mathrm{M}$ & $\mathrm{L}$ & $\mathrm{M}$ \\
$C_{12}$ & $\mathrm{H}$ & $\mathrm{VH}$ & $\mathrm{VH}$ & $\mathrm{H}$ \\
$C_{13}$ & $\mathrm{H}$ & $\mathrm{M}$ & $\mathrm{H}$ & $\mathrm{M}$ \\
$C_{14}$ & $\mathrm{H}$ & $\mathrm{M}$ & $\mathrm{H}$ & $\mathrm{H}$ \\
\hline
\end{tabular}

The original decision matrix is identified by the raters by observing the websites, and the normalized decision matrix is then derived from the original data as shown in Table 6 .

The larger, the better type:

$$
r_{i j}=\frac{\left[x_{i j}-\min \left\{x_{i j}\right\}\right]}{\left[\max \left\{x_{i j}\right\}-\min \left\{x_{i j}\right\}\right]}
$$

The smaller, the better type:

$$
r_{i j}=\frac{\left[\max \left\{x_{i j}\right\}-x_{i j}\right]}{\left[\max \left\{x_{i j}\right\}-\min \left\{x_{i j}\right\}\right]}
$$


Table 6. Part of the Normalized Decision Matrix

\begin{tabular}{lllllllllllll}
\hline No. & $C_{1}$ & $C_{2}$ & $C_{3}$ & $C_{4}$ & $C_{5}$ & $C_{6}$ & $C_{7}$ & $C_{8}$ & $C_{9}$ & $C_{10}$ & $C_{11}$ & $C_{12}$ \\
\hline$E_{1}$ & 0.9 & 0.8 & 0.8 & 0.9 & 0.9 & 0.8 & 0.8 & 0.9 & 0.8 & 0.8 & 0.8 & 0.9 \\
$E_{2}$ & 0.9 & 0.9 & 0.9 & 0.9 & 0.9 & 1 & 0.6 & 0.8 & 0.7 & 0.7 & 0.8 & 0.9 \\
$E_{3}$ & 0.9 & 0.9 & 0.9 & 0.8 & 0.8 & 0.9 & 0.8 & 0.8 & 0.8 & 0.7 & 0.9 & 0.8 \\
$E_{4}$ & 0.8 & 0.8 & 0.6 & 0.7 & 0.8 & 0.7 & 0.8 & 0.7 & 0.8 & 0.8 & 0.7 & 0.7 \\
$E_{5}$ & 0.7 & 0.7 & 0.9 & 0.8 & 0.6 & 0.8 & 0.6 & 0.5 & 0.7 & 0.4 & 0.8 & 0.4 \\
$E_{6}$ & 0.8 & 0.9 & 0.6 & 0.6 & 0.8 & 0.9 & 0.8 & 0.8 & 0.8 & 0.7 & 0.7 & 0.8 \\
$E_{7}$ & 0.8 & 0.6 & 0.5 & 0.8 & 0.8 & 0.8 & 0.7 & 0.7 & 0.6 & 0.7 & 0.5 & 0.8 \\
$E_{8}$ & 0.8 & 0.8 & 0.8 & 0.8 & 0.7 & 0.7 & 0.8 & 0.8 & 0.6 & 0.7 & 0.7 & 0.8 \\
$E_{9}$ & 0.8 & 0.8 & 0.6 & 0.8 & 0.8 & 0.9 & 0.6 & 0.8 & 0.8 & 0.5 & 0.7 & 0.8 \\
$E_{10}$ & 0.7 & 0.9 & 0.6 & 0.8 & 0.8 & 0.9 & 0.8 & 0.8 & 0.8 & 0.7 & 0.8 & 0.7 \\
$E_{11}$ & 0.6 & 0.8 & 0.8 & 0.9 & 0.8 & 0.7 & 0.6 & 0.5 & 0.7 & 0.8 & 0.6 & 0.6 \\
$E_{12}$ & 0.5 & 0.5 & 0.8 & 0.6 & 0.8 & 0.6 & 0.5 & 0.7 & 0.8 & 0.4 & 0.7 & 0.7 \\
$E_{13}$ & 0.6 & 0.8 & 0.6 & 0.7 & 0.7 & 0.8 & 0.6 & 0.8 & 0.7 & 0.6 & 0.6 & 0.5 \\
$E_{14}$ & 0.5 & 0.4 & 0.8 & 0.6 & 0.8 & 0.7 & 0.7 & 0.8 & 0.5 & 0.4 & 0.8 & 0.6 \\
$E_{15}$ & 0.8 & 0.7 & 0.6 & 0.8 & 0.8 & 0.7 & 0.8 & 0.7 & 0.6 & 0.6 & 0.6 & 0.8 \\
\hline & & & & & & & & & & & &
\end{tabular}

Then the normalized decision matrix using Fuzzy linguistic variables shown in Table 7 can be identified by the Fuzzy membership function proposed in Section 3.2.

Table 7. Part of the Normalized Decision Matrix using Fuzzy Linguistic Variables

\begin{tabular}{lllllllllllll}
\hline No. & $C_{1}$ & $C_{2}$ & $C_{3}$ & $C_{4}$ & $C_{5}$ & $C_{6}$ & $C_{7}$ & $C_{8}$ & $C_{9}$ & $C_{10}$ & $C_{11}$ & $C_{12}$ \\
\hline$E_{1}$ & $\mathrm{VH}$ & $\mathrm{H}$ & $\mathrm{H}$ & $\mathrm{VH}$ & $\mathrm{VH}$ & $\mathrm{H}$ & $\mathrm{H}$ & $\mathrm{VH}$ & $\mathrm{H}$ & $\mathrm{H}$ & $\mathrm{H}$ & $\mathrm{VH}$ \\
$E_{2}$ & $\mathrm{VH}$ & $\mathrm{VH}$ & $\mathrm{VH}$ & $\mathrm{VH}$ & $\mathrm{VH}$ & $\mathrm{VH}$ & $\mathrm{M}$ & $\mathrm{H}$ & $\mathrm{H}$ & $\mathrm{H}$ & $\mathrm{H}$ & $\mathrm{VH}$ \\
$E_{3}$ & $\mathrm{VH}$ & $\mathrm{VH}$ & $\mathrm{VH}$ & $\mathrm{H}$ & $\mathrm{H}$ & $\mathrm{VH}$ & $\mathrm{H}$ & $\mathrm{H}$ & $\mathrm{H}$ & $\mathrm{H}$ & $\mathrm{VH}$ & $\mathrm{H}$ \\
$E_{4}$ & $\mathrm{H}$ & $\mathrm{H}$ & $\mathrm{M}$ & $\mathrm{H}$ & $\mathrm{H}$ & $\mathrm{H}$ & $\mathrm{H}$ & $\mathrm{H}$ & $\mathrm{H}$ & $\mathrm{H}$ & $\mathrm{H}$ & $\mathrm{H}$ \\
$E_{5}$ & $\mathrm{H}$ & $\mathrm{H}$ & $\mathrm{VH}$ & $\mathrm{H}$ & $\mathrm{M}$ & $\mathrm{H}$ & $\mathrm{M}$ & $\mathrm{M}$ & $\mathrm{H}$ & $\mathrm{L}$ & $\mathrm{H}$ & $\mathrm{L}$ \\
$E_{6}$ & $\mathrm{H}$ & $\mathrm{VH}$ & $\mathrm{M}$ & $\mathrm{M}$ & $\mathrm{H}$ & $\mathrm{VH}$ & $\mathrm{H}$ & $\mathrm{H}$ & $\mathrm{H}$ & $\mathrm{H}$ & $\mathrm{H}$ & $\mathrm{H}$ \\
$E_{7}$ & $\mathrm{H}$ & $\mathrm{M}$ & $\mathrm{M}$ & $\mathrm{H}$ & $\mathrm{H}$ & $\mathrm{H}$ & $\mathrm{H}$ & $\mathrm{H}$ & $\mathrm{M}$ & $\mathrm{H}$ & $\mathrm{M}$ & $\mathrm{H}$ \\
$E_{8}$ & $\mathrm{H}$ & $\mathrm{H}$ & $\mathrm{H}$ & $\mathrm{H}$ & $\mathrm{H}$ & $\mathrm{H}$ & $\mathrm{H}$ & $\mathrm{H}$ & $\mathrm{M}$ & $\mathrm{H}$ & $\mathrm{H}$ & $\mathrm{H}$ \\
$E_{9}$ & $\mathrm{H}$ & $\mathrm{H}$ & $\mathrm{M}$ & $\mathrm{H}$ & $\mathrm{H}$ & $\mathrm{VH}$ & $\mathrm{M}$ & $\mathrm{H}$ & $\mathrm{H}$ & $\mathrm{M}$ & $\mathrm{H}$ & $\mathrm{H}$ \\
$E_{10}$ & $\mathrm{H}$ & $\mathrm{VH}$ & $\mathrm{M}$ & $\mathrm{H}$ & $\mathrm{H}$ & $\mathrm{VH}$ & $\mathrm{H}$ & $\mathrm{H}$ & $\mathrm{H}$ & $\mathrm{H}$ & $\mathrm{H}$ & $\mathrm{H}$ \\
$E_{11}$ & $\mathrm{M}$ & $\mathrm{H}$ & $\mathrm{H}$ & $\mathrm{VH}$ & $\mathrm{H}$ & $\mathrm{H}$ & $\mathrm{M}$ & $\mathrm{M}$ & $\mathrm{H}$ & $\mathrm{H}$ & $\mathrm{M}$ & $\mathrm{M}$ \\
$E_{12}$ & $\mathrm{M}$ & $\mathrm{M}$ & $\mathrm{H}$ & $\mathrm{M}$ & $\mathrm{H}$ & $\mathrm{M}$ & $\mathrm{M}$ & $\mathrm{H}$ & $\mathrm{H}$ & $\mathrm{L}$ & $\mathrm{H}$ & $\mathrm{H}$ \\
$E_{13}$ & $\mathrm{M}$ & $\mathrm{H}$ & $\mathrm{M}$ & $\mathrm{H}$ & $\mathrm{H}$ & $\mathrm{H}$ & $\mathrm{M}$ & $\mathrm{H}$ & $\mathrm{H}$ & $\mathrm{M}$ & $\mathrm{M}$ & $\mathrm{M}$ \\
$E_{14}$ & $\mathrm{M}$ & $\mathrm{L}$ & $\mathrm{H}$ & $\mathrm{M}$ & $\mathrm{H}$ & $\mathrm{H}$ & $\mathrm{H}$ & $\mathrm{H}$ & $\mathrm{M}$ & $\mathrm{L}$ & $\mathrm{H}$ & $\mathrm{M}$ \\
$E_{15}$ & $\mathrm{H}$ & $\mathrm{H}$ & $\mathrm{M}$ & $\mathrm{H}$ & $\mathrm{H}$ & $\mathrm{H}$ & $\mathrm{H}$ & $\mathrm{H}$ & $\mathrm{M}$ & $\mathrm{M}$ & $\mathrm{M}$ & $\mathrm{H}$ \\
\hline
\end{tabular}

The Fuzzy linguistic variable is then transformed into a Fuzzy triangular membership function as shown in Table 8, and then the resulting Fuzzy weighted decision matrix can be derived based on Table 8 and the weights identified before. The distance of each alternative from $A^{+}$and $A^{-}$, as well as the similarities to an ideal solution $(C C i)$, is obtained in Table 9. 
Table 8. Part of the Fuzzy Decision Matrix

\begin{tabular}{lccccc}
\hline & $C_{1}$ & $C_{2}$ & $C_{3}$ & $C_{4}$ & $C_{5}$ \\
\hline$E_{1}$ & $(0.75,0.90,1.00)$ & $(0.55,0.70,0.85)$ & $(0.55,0.70,0.85)$ & $(0.75,0.90,1.00)$ & $(0.75,0.90,1.00)$ \\
$E_{2}$ & $(0.75,0.90,1.00)$ & $(0.75,0.90,1.00)$ & $(0.75,0.90,1.00)$ & $(0.75,0.90,1.00)$ & $(0.75,0.90,1.00)$ \\
$E_{3}$ & $(0.75,0.90,1.00)$ & $(0.75,0.90,1.00)$ & $(0.75,0.90,1.00)$ & $(0.55,0.70,0.85)$ & $(0.55,0.70,0.85)$ \\
$E_{4}$ & $(0.55,0.70,0.85)$ & $(0.55,0.70,0.85)$ & $(0.35,0.50,0.65)$ & $(0.55,0.70,0.85)$ & $(0.55,0.70,0.85)$ \\
$E_{5}$ & $(0.55,0.70,0.85)$ & $(0.55,0.70,0.85)$ & $(0.75,0.90,1.00)$ & $(0.55,0.70,0.85)$ & $(0.35,0.50,0.65)$ \\
$E_{6}$ & $(0.55,0.70,0.85)$ & $(0.75,0.90,1.00)$ & $(0.35,0.50,0.65)$ & $(0.35,0.50,0.65)$ & $(0.55,0.70,0.85)$ \\
$E_{7}$ & $(0.55,0.70,0.85)$ & $(0.35,0.50,0.65)$ & $(0.35,0.50,0.65)$ & $(0.55,0.70,0.85)$ & $(0.55,0.70,0.85)$ \\
$E_{8}$ & $(0.55,0.70,0.85)$ & $(0.55,0.70,0.85)$ & $(0.55,0.70,0.85)$ & $(0.55,0.70,0.85)$ & $(0.55,0.70,0.85)$ \\
$E_{9}$ & $(0.55,0.70,0.85)$ & $(0.55,0.70,0.85)$ & $(0.35,0.50,0.65)$ & $(0.55,0.70,0.85)$ & $(0.55,0.70,0.85)$ \\
$E_{10}$ & $(0.55,0.70,0.85)$ & $(0.75,0.90,1.00)$ & $(0.35,0.50,0.65)$ & $(0.55,0.70,0.85)$ & $(0.55,0.70,0.85)$ \\
$E_{11}$ & $(0.35,0.50,0.65)$ & $(0.55,0.70,0.85)$ & $(0.55,0.70,0.85)$ & $(0.75,0.90,1.00)$ & $(0.55,0.70,0.85)$ \\
$E_{12}$ & $(0.35,0.50,0.65)$ & $(0.35,0.50,0.65)$ & $(0.55,0.70,0.85)$ & $(0.35,0.50,0.65)$ & $(0.55,0.70,0.85)$ \\
$E_{13}$ & $(0.35,0.50,0.65)$ & $(0.55,0.70,0.85)$ & $(0.35,0.50,0.65)$ & $(0.55,0.70,0.85)$ & $(0.55,0.70,0.85)$ \\
$E_{14}$ & $(0.35,0.50,0.65)$ & $(0.15,0.30,0.45)$ & $(0.55,0.70,0.85)$ & $(0.35,0.50,0.65)$ & $(0.55,0.70,0.85)$ \\
$E_{15}$ & $(0.55,0.70,0.85)$ & $(0.55,0.70,0.85)$ & $(0.35,0.50,0.65)$ & $(0.55,0.70,0.85)$ & $(0.55,0.70,0.85)$ \\
$\otimes W$ & $(0.55,0.70,0.84)$ & $(0.65,0.80,0.93)$ & $(0.30,0.45,0.60)$ & $(0.50,0.65,0.80)$ & $(0.50,0.65,0.80)$ \\
\hline
\end{tabular}

Table 9. The Distance of each Alternative from $A^{+}$and $A$

\begin{tabular}{llll}
\hline E-Commerce & $d_{i}^{+}$ & $d_{i}^{-}$ & $C C i$ \\
\hline Tmall & 0.03412 & 0.204884 & 0.857241 \\
Jingdong Mall & 0.023991 & 0.214935 & 0.899587 \\
Tencent & 0.049565 & 0.189531 & 0.792697 \\
Amazon & 0.100974 & 0.13825 & 0.577909 \\
Handle group buying & 0.166283 & 0.072738 & 0.304316 \\
Dangdang & 0.094963 & 0.14412 & 0.602804 \\
Vancl & 0.106483 & 0.132646 & 0.554705 \\
Full King & 0.090915 & 0.148385 & 0.620079 \\
No.1 & 0.107855 & 0.13134 & 0.549092 \\
F group buying & 0.085558 & 0.153569 & 0.642207 \\
Yixun & 0.124634 & 0.114415 & 0.478625 \\
Moonbasa & 0.1512 & 0.087884 & 0.367587 \\
Letao & 0.140376 & 0.09872 & 0.412889 \\
Newegg & 0.188149 & 0.05084 & 0.212731 \\
M18 & 0.13909 & 0.100051 & 0.418375 \\
\hline
\end{tabular}

\section{Conclusions}

This paper is an attempt to identify the main factors affecting the online service satisfaction of the e-commerce websites in China, and Fuzzy TOPSIS is employed to evaluate the e-service quality through internet for the online consumers. Considering the differences among $\mathrm{B} 2 \mathrm{C}, \mathrm{C} 2 \mathrm{C}$ and $\mathrm{B} 2 \mathrm{~B}$ e-commerce, and this research is focus on $\mathrm{B} 2 \mathrm{C}$ e-commerce websites in China, the top 15 B2C e-commerce websites in retail market are selected based on the user coverage.

According to the result of this research, there exist large gap among the selected top 15 B2C e-commerce websites in China in terms of the e-service quality, for example, the highest score among these e-commerce websites is 0.899587 , whereas the lowest scores is 0.212731 . The top three e-commerce websites (Tmall, Jingdong Mall and Tencent) have the relatively better performance than others. According to the criteria weights derived from this section earlier, the suggestions for these e-commerce websites to improve their e-service are: (1) the e-commerce companies shall improve the customer service to 
excellent standards; (2) the e-commerce companies shall focus on flexibility and responsiveness to be able to adapt to market changes; (3) the e-commerce companies shall focus on efficiency to make sure the online shoppers can search the goods they wanted in short time; (4) the e-commerce companies shall improve the assurance to absorb more online shoppers and (5) the e-commerce companies should promote ease of use through better user interface design on the websites.

\section{References}

[1] S. Gounaris and S. Dimitriadis, "Assessing service quality on the web: evidence from business-toconsumer portals", Journal of Services Marketing, vol. 17, no. 4-5, (2003), pp. 529-548.

[2] S. Jarvenpaa and P. Todd, "Consumer reactions to electronic shopping on the world wide web", International Journal of Electronic Commerce, vol. 1, no. 2, (1997), pp. 59-88.

[3] D. Gefen, "Customer loyaltyine-commerce", Journal of the Association for Information Systems, vol. 3, (2002), pp. 27-51.

[4] Y. Hwang and D. J. Kim, "Customer self-service systems: the effects of perceived web quality with service contents on enjoyment, anxiety, ande-trust", Decision Support Systems, vol. 43, (2007), pp. 746760 .

[5] S. H. Hsu, "Developing an index for online customer satisfaction: adaptation of American customer satisfaction index", Expert Systems with Applications, vol. 34, (2008), pp. 3033-3042.

[6] S. Ha and L. Stoel, "Consumer e-shopping acceptance: antecedents in a technology acceptance model", Journal of Business Research, vol. 62, (2009), pp. 565-571.

[7] C. I. Ho and Y. L. Lee, "The development of an e-travel service quality scale", Tourism Management, vol. 26, (2007), pp. 1434-1449.

[8] B. Yoo and N. Donthu, "Developing a scale to measure the perceived quality of Internet shopping sites (SITEQUAL)", Quarterly Journal of Electronic Commerce, vol. 2, no. 1, (2001), pp. 31-47.

[9] G. Lee and H. Lin, "Customer perceptions of e-service quality in onlines hopping", International Journal of Retail and Distribution Management, vol. 33, no. 2, (2005), pp. 161-176.

[10] E. Cristobal, C. Flavian and M. Guinaliu, "Perceived e-service quality (PeSQ): measurement validation and effects on consumer satisfaction and website loyalty", Managing Service Quality, vol. 17, no. 3, (2007), pp. 317-340.

[11] V. A. Zeithaml, A. Parasuraman and A. Malhotra, "Service quality delivery through websites: acritical review of extant knowledge", Journal of the Academy of Marketing Science, vol. 30, no. 4, (2002), pp. $362-375$.

[12] J. Santos, "E-service quality: a model of virtual service quality dimension", Managing Service Quality, vol. 13 , no. 3, (2003), pp. 233-246.

[13] M. Wolfinbarger and M. C. Gilly, "ETailQ: dimensionalizing, measuring and predicting retail quality", Journal of Retailing, vol. 79, no. 3, (2003), pp. 183-198.

[14] B. B. Holloway and S. E. Beatty, "Service failure in online retailing: a recovery opportunity", Journal of Service Research, vol. 6, no. 1, (2003), pp. 92-105.

[15] M. Howard and C. Worboys, "Self-service - a contradiction in terms or customer-led choice?", Journal Consume Behavior, vol. 2, no. 4, (2003), pp. 382-92.

[16] V. A. Zeithaml, A. Parasuraman and A. Malhotra, "E-service quality: definition, dimensions and conceptual model", Working Paper, Marketing Science Institute, (2000).

[17] A. Parasuraman, V. A. Zeithaml A. Malhotra, "E-S-Qual: a multiple-item scale for assessing electronic service quality", Journal of Service Research, vol. 7, no. 3, (2005), pp. 213-233.

[18] A. Serkan, A. Eda and A. Safak, "Re-assessment of E-S-Qual and E-RecS-Qual in a pure service setting", Journal of Business Research, vol. 63, no. 3, (2010), pp. 232-240.

[19] E. T. Loiacono, R. T. Watson and D. L. Hoodhue, "WEBQUAL:measure of website quality", Marketing Educators Conference: Marketing Theory and Applications, (2002).

[20] B. Grégory, "A consumer typology based on e-service quality and e-satisfaction”, Journal of Retailing and Consumer Services, vol. 21, no. 6, (2014), pp. 889-896.

[21] H. H. Bauer, T. Falk and M. Hammerschmidt, "E Trans Qual:a transaction process-based approach for capturing service quality in online shopping", Journal of Business Research, vol. 59, (2006), pp. 866-875.

[22] B. B. Ramón, "Evaluation of the e-service quality in service encounters with incidents: Differences according to the socio-demographic profile of the online consumer", Revista Europea de Dirección y Economía de la Empresa, vol. 23, no. 4, (2014), pp. 184-193.

[23] R. R. Nelson, P. A. Todd and B. H. Wixom, "Antecedents of information and system quality: an empirical examination within the context of data warehousing", Journal of Management Information Systerm, vol. 21, no. 4, (2005), pp. 199-235.

[24] B. H. Wixom and P. A. Todd, "A theoretical integration of user satisfaction and technology acceptance?", Information System Research, vol. 16, no. 1, (2005), pp. 85-102. 
[25] T. Hamed, "Features' evaluation of goods, services and e-services; electronic service characteristics exploration", The 7th International Conference Interdisciplinarity in Engineering, (2014).

[26] J. E. Francis and L. White, "PIRQUAL: a scale for measuring customer expectations and perceptions of quality in Internet retailing", Proceedings of the Winter Educator's Conference, AMA, Chicago, IL: American Marketing Association, (2002).

[27] S. J. Barnes and R. T. Vidgen, "An integrative approach to the assessment of e-commerce quality", Journal of Electronic Commerce Research, vol. 3, no. 3, (2002), pp. 114-27.

[28] A. M. Aladwani and P. C. Palvia, "Developing and validating an instrument for measuring userperceived web quality", Information and Management, vol. 39, no. 6, (2002), pp. 467-476.

[29] S. Janda, P. J. Trocchia and K. P. Gwinner, "Consumer perceptions of Internet retail service quality", International Journal of Service Industry Management, vol. 13, no. 5, (2002), pp. 412-431.

[30] C. Ranganathan and S. Ganapathy, "Key dimensions of business-to-consumer web sites", Information and Management, vol. 39, (2002), pp. 457-465.

[31] Z. Yang and M. Jun, "Consumer perception of e-service quality: form purchaser and non purchaser perspectives", Journal of Business Strategies, vol. 19, no. 1, (2002), pp. 19-41.

[32] S. Cai and M. Jun, "Internetusers'perceptions of online service quality: a comparison of online buyers and information searchers", Managing Service Quality, vol. 13, no. 6, (2003), pp 504-519.

[33] M. Jun, Z. Yang and D. Kim, "Customers' perceptions of online retailing service quality and their satisfaction", International Journal of Quality and Reliability Management, vol. 21, no. 8, (2004), pp. 817-840.

[34] S. Kim and L. Stoel, “Apparel retailers: website quality dimensions and satisfaction”, Journal of Retailing and Consumer Services, vol. 11, no. 2, (2004), pp. 109-117

[35] Z. Yang, "Development and validation of an instrument to measure user perceived service quality of information presenting Web portals", Information and Management, vol. 42, (2005), pp. 575-589.

[36] J. E. Collier and C. C. Bienstock, "Measuring service quality in e-retailing", Journal of Service Research, vol. 8, no. 3, (2006), pp. 260-275.

[37] E. E. Ibrahim, M. Joseph and K. I. N. Ibeh, "Customers'perception of electronic service delivery in the UK retail banking sector", International Journal of Bank Marketing, vol. 24, no. 7, (2006), pp. 475-493.

[38] C. Sohn and S. K. Tadisina, "Development of e-service quality measure for internet-based financial institutions", Total Quality Management and Business Excellence, vol. 19, no. 9, (2008), pp. 903-918.

[39] Y. Wang, D. M. Hernandez and S. M. Minor, "Web aesthetics effects on perceived online service quality and satisfaction in an e-tail environment: the moderating role of purchase task", Journal of Business Research, vol. 63, (2010), pp. 935-942.

[40] D. X. Ding, J. P. Hu and L. O. Sheng, "E-SELFQUAL: a scale for measuring online self-service quality", Journal of Business Research, vol. 64, (2011), pp. 508-515.

\section{Author}

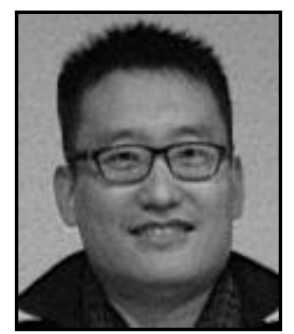

Zhenfeng Wei was born and raised in Shandong Province of China. He graduated from Tongji University with a master's degree of Computer Engineering in June, 2010. Now he is a lecturer of Zhejiang Industry \& Trade Vocational College. His current research interests include Electronic Commerce, Online Marketing and Mobile Electronic Commerce. 
International Journal of $\mathrm{u}-$ and $\mathrm{e}-$ Service, Science and Technology Vol.8, No.3 (2015) 